\title{
COVID-19 and Alcohol - A Review
}

\section{Supriya Garapati ${ }^{1}$, Lakshmi Jyothi Tadi ${ }^{2 *}$, Ariyanachi $\mathrm{K}^{3}$ and Pravin $\mathrm{P}^{4}$}

${ }^{1}$ Associate Professor, Department of Anatomy, All India Institute of Medical Sciences, Bibinagar, India

${ }^{2}$ Additional Professor, Department of Microbiology, All India Institute of Medical

Sciences, Bibinagar, India

${ }^{3}$ Assistant Professor, Department of Anatomy, All India Institute of Medical Sciences, Bibinagar, India

${ }^{4}$ Associate Professor, Department of Community Medicine, ESIC Medical College and Hospital, Hyderabad, India

*Corresponding Author: Lakshmi Jyothi Tadi, Additional Professor, Department of Microbiology, All India Institute of Medical Sciences, Bibinagar, India.
Received: August 19,2021

Published: September 22, 2021

(C) All rights are reserved by Lakshmi Jyothi

Tadi., et al.

\section{Abstract}

Background: Alcohol use has been associated to a number of disorders, including SARS-CoV-2 infection since Alcohol can have a negative impact on the immune system through multiple pathophysiological mechanisms like reducing the number of $\mathrm{T}$ lymphocytes, favoring a pro-inflammatory status through an increased level of proinflammatory cytokines, such as tumor necrosis factor alfa (TNF $\alpha$ ) and interleukins 1 and 6 (IL-1, IL-6), by decreasing the function, number of NK (Natural Killers) cells responsible for removing infected or malignant cells, disturbing the macrophage functions in the lung alveoli and damage to the respiratory ciliated cells which plays an essential role in filtering pathogenic microorganisms. It can also worsen mental and biological conditions, as well as predispose to behaviour that increase the risk of infection and disease severity, though not necessarily sociopathic or violent behaviour. Another important factor is malnutrition secondary to excessive alcohol intake.

Introduction: Alcohol misuse impairs both the innate and adaptive immune systems, potentially lowering resistance to the coronavirus SARS-CoV-2 and speeding up the onset of coronavirus illness (COVID-19). Alcohol use has been associated to several disorders, including SARS-CoV-2 infection since Alcohol can have a negative impact on the immune system through multiple pathophysiological mechanisms.

Aims and Objectives

- $\quad$ To review the relationship between Covid-19 and alcoholism

- $\quad$ To learn about the illness signs of alcohol use in Covid-19

- To compare the mortality of alcoholics and non-alcoholics in the covid-19 population.

- To learn about the effects of alcohol withdrawal in the aftermath of the pandemic.

Methodology: Data was gathered from PubMed Central, Medscape, NCBI's website, PMC, and BMJ journals, among other sources. The data on the relationship between alcohol and Covid-19 has been examined, analysed, and summarised in this publication. 
Review: When it comes to COVID-19, patients with alcohol use disorder or alcohol-related liver disease, as well as relevant comorbidities, are at a higher risk.

The World Health Organization (World Health Organization, 2020) [26] and the National Institute on Alcohol Abuse and Alcoholism (National Institute on Alcohol Abuse and Alcoholism, 2020) [27] have issued comments stating that heavy alcohol consumption does not prevent COVID-19 infection.

Mucociliary clearance is regarded to be the first line of defence against inhaled pathogenic microorganisms, even though alcohol has long been known to decrease coughing (Calesnick and Vernick, 1971). Heavy drinking disrupts the cilia's cyclic nucleotide pathway, limiting cilia beat frequency from increasing (Wyatt and Sisson, 2001) [28].

Discussion: Alcohol raises the risk of death and worsens the symptoms of ARDS. By increasing alveolar barrier permeability, alcohol consumption affects the oxidative stress response and makes the lungs more sensitive to oedematous stress. AUD is often associated with comorbidities such as diabetes mellitus and chronic kidney disease, which also increase the risk for complications in COVID-19 [34].

Conclusion: Alcohol influences practically every lung cell. Most of these modifications may increase the likelihood of COVID-19 infection and more severe pneumonia or ARDS in heavy drinkers.

Keywords: Alcohol; Covid-19; SARS-CoV-2; ARDS (Acute Respiratory Distress Syndrome)

\section{Abbreviations}

SARS-CoV-2; TNF $\alpha$; IL-1; IL-6; SARS; MERS; AUD: Alcohol Use Disorder; AW: Alcohol Withdrawal.

\section{Introduction}

Coronaviruses cause respiratory infections in humans, which can range from a simple cold to life-threatening diseases like Middle East Respiratory Syndrome (MERS) and severe acute respiratory syndrome (SARS) [1]. COVID-19 disease is caused by the coronavirus SARS-CoV-2 [2]. COVID-19 was initially discovered in individuals with pneumonia in late 2019 in Wuhan, China's capital and capital of Hubei Province [3].

Excessive alcohol use impairs the immune system, making it more susceptible to infection with the SARS-CoV-2 virus in the current setting.

Millions of individuals die prematurely because of excessive alcohol use around the world. The new coronavirus has been associated to a number of medical and mental problems [4], all of which can make a person prone to it. Because alcohol weakens the immune system, it increases the chance of contracting certain diseases [5]. AUD is often associated with comorbidities such as dia- betes mellitus and chronic kidney disease, which also increase the risk for complications in COVID-19 [34].

COVID-19 may affect alcohol consumption in two ways, physical and financial availability according to specialists in the field [6].

Alcohol is a widely available chemical that is widely tolerated in most communities, yet it has the potential for addiction as well as acute and chronic toxicity [7]. It depresses the central nervous system, resulting in a reduction in neuronal activity [8-10]. Many patients who suffer from anxiety, sadness, or sleeplessness utilise it because of its anxiolytic, antidepressant, relaxing, and sedative properties [11].

Acute and chronic ethanol poisoning can affect the entire body [12], causing general medical disorders because of alcohol usage.

\section{Aims and Objectives}

- To review the relationship between Covid-19 and alcoholism

- $\quad$ To learn about the illness signs of alcohol use in Covid-19

- $\quad$ To review the mortality of alcoholics and non-alcoholics in the covid-19 population. 
- To learn about the effects of alcohol withdrawal in the aftermath of the pandemic.

\section{Materials and Methods}

Data was gathered from PubMed Central, Medscape, NCBI's website, PMC, and BMJ journals, among other sources. Around 32 articles were reviewed. The data on the relationship between alcohol and Covid-19 has been examined, analysed, and summarised in this publication.

\section{Review}

Around 30 articles from various databases were compiled and reviewed.

\section{Results and Discussion}

A survey of 1,982 people in the United States found that for every week spent at home in isolation during the pandemic, the likelihood of an alcoholic engaging in binge drinking increased by 1.19 percent at the conclusion of the week. The survey also revealed that binge drinkers consume $60 \%$ more alcohol than occasional drinkers, with 69 percent of female binge drinkers and 31 percent of male binge drinkers [13].

When compared to pre-COVID-19, another cross-sectional U.S. survey of 832 participants found that $60 \%$ reported increased drinking and $13 \%$ reported decreased drinking during the pandemic. More stress (45.7\%), increased alcohol availability (34.4\%), and boredom were all cited as reasons for increased drinking (30.1 percent). Participants who said they were stressed by the pandemic drank more alcohol over a longer period of time, raising implications for both individual and public health [14].

Almost every organ in your body is affected by alcohol, whether short- and long-term. Overall, the research implies that there is no such thing as a "safe limit" for alcohol consumption; in fact, the risk of harm to one's health grows with each drink drank. Alcohol abuse, particularly heavy drinking, impairs the immune system, reducing the body's ability to fight infectious infections [15].

Alcohol use would have aided in the elimination of the SARSCoV-2 virus, according to current incorrect information. This notion is based on no scientific evidence; in fact, alcohol intake weakens the body's defences against viral respiratory diseases [16].
Although there is no specific guidance on management of alcoholic hepatitis during the COVID-19 pandemic, caution is advised regarding glucocorticoids therapy in SARS-CoV-2 infection [34].

The following topics are addressed in WHO's published guidelines on maintaining a balanced psychological state [17].

According to the findings of a cross-sectional survey conducted in Romania on 115 male patients and 57 controls, patients with severe mental illness and alcohol use disorder are at an increased risk of getting COVID-19 and having poor COVID-19 infection outcomes. One factor is a lack of knowledge of COVID-19 prevention methods and an inability to distinguish false information from reality [18].

Ram A. Sharma, MD., et al. [19] investigated the link between the COVID-19 pandemic and AUD (Alcohol Use Disorder) and AW (Alcohol withdrawal). Because greater rates of AW persisted during the reopening phases, stress, worry, interrupted treatment plans, and increased alcohol consumption could all be contributors. He also objectively identified the pandemic's harmful effects on alcohol withdrawal: "In 2020, there were 34\% greater alcohol withdrawal rates among hospitalised patients than in 2019." since the Alcohol can induce alterations in various pathophysiological mechanisms, withdrawal might help reducing the negative impact. AUD is often associated with comorbidities such as diabetes mellitus and chronic kidney disease, which also increase the risk for complications in COVID-19 [34].

Prior to the stay-at-home order, 340 patients were diagnosed with alcohol withdrawal, 231 patients were diagnosed during the stay-at-home period, and 507 patients were diagnosed following the stay-at-home period. In 2020, hospitalised patients experienced a higher rate of alcohol withdrawal than in 2019 and the combined average of 2018 and 2019; however, the difference was bigger in the period after the stay-at-home order. The incidence rate ratio was higher in 2020 compared to 2019 in the latter two weeks of the stay-at-home order. Alcohol withdrawal rates among hospitalised patients increased by 34\% during the pandemic in 2020, compared to the same period in 2019.

When it comes to COVID-19, patients with alcohol use disorder or alcohol-related liver disease, as well as relevant comorbidities, are at a higher risk [20]. 
In the research, two distinct scenarios for how COVID-19 pandemic lockdowns might affect alcohol consumption have been proposed [21]. Second, psychological distress mechanisms such as fear of infection, unemployment, or social isolation can generate stress and anxiety, leading to increased alcohol consumption [22].

Little is known about how excessive alcohol use affects COVID-19 infection. Despite the fact that less than 15\% of drunk alcohol enters the lungs, exhalation is a substantial process for alcohol excretion, as indicated by the widespread use of the breathalyser test to accurately assess blood alcohol levels (Birkenstein and Smith, 1961) [23]. Heavy alcohol use alters pulmonary innate and adaptive immunity, increasing the risk of viral pneumonia (de Roux., et al. 2006), influenza A infection (Wyatt., et al. 2019), respiratory syncytial virus (RSV) infection (Wyatt., et al. 2019), and bacterial pneumonia (Wyatt., et al. 2019).

In Iran, there was a popular belief that gargling with alcohol or consuming alcoholic beverages would disinfect the mouth or the inside of the body, preventing infection by killing the virus. The World Health Organization (World Health Organization, 2020) [26] and the National Institute on Alcohol Abuse and Alcoholism (National Institute on Alcohol Abuse and Alcoholism, 2020) [27] have issued comments stating that heavy alcohol consumption does not prevent COVID-19 infection.

Mucociliary clearance is regarded to be the first line of defence against inhaled pathogenic microorganisms, despite the fact that alcohol has long been known to decrease coughing (Calesnick and Vernick, 1971). The major airways are lined with ciliated and mucus-producing airway epithelial cells. To push mucus containing trapped particles out of the lung and into the oropharynx, Cilia beat in tandem. Heavy drinking disrupts the cilia's cyclic nucleotide pathway, limiting cilia beat frequency from increasing (Wyatt and Sisson, 2001) [28]. Protein kinase C epsilon, an enzyme that is elevated in the ciliated cells of the airway in older persons, may actively block the cilia if the heavy drinker also smokes cigarettes, which is common (Bailey, Kharbanda, Katafiasz, Sisson, and Wyatt, 2018) [29].

COVID-19 is linked to COPD, according to an early meta-analysis of individual findings. COPD is, in fact, the single most important predictor of comorbidity for severe COVID-19 requiring ICU hospitalisation (Jain and Yuan, 2020) [30]. However, smokers are more likely to consume alcohol, and drinking has been found to be an independent risk factor for COPD (Nihlen, Greiff, Nyberg, Persson, and Andersson, 2005) [31].

Alcohol raises the risk of death and worsens the symptoms of ARDS. By increasing alveolar barrier permeability, alcohol consumption affects the oxidative stress response and makes the lungs more sensitive to oedematous stress (Esper, Burnham, and Moss, 2006) [32].

Xiude Fan., et al. [33] discovered that frequent drinking, especially heavy drinking, was connected to an elevated risk of death in people with obesity and COVID-19, but not in people who did not have obesity.

\section{Discussion}

The World Health Organization (World Health Organization, 2020) [26] and the National Institute on Alcohol Abuse and Alcoholism (National Institute on Alcohol Abuse and Alcoholism, 2020) [27] have issued comments stating that heavy alcohol consumption does not prevent COVID-19 infection. Heavy alcohol use alters pulmonary innate and adaptive immunity, increasing the risk of pneumoniae of viral, bacterial and fungal etiology. Alcohol can have a negative impact on the immune system through multiple pathophysiological mechanisms like reducing the number of $\mathrm{T}$ lymphocytes, favoring a pro-inflammatory status through an increased level of proinflammatory cytokines, such as tumor necrosis factor alfa (TNF $\alpha$ ) and interleukins 1 and 6 (IL-1, IL-6), by decreasing the function, number of NK (Natural Killers) cells responsible for removing infected or malignant cells, disturbing the macrophage functions in the lung alveoli and damage to the respiratory ciliated cells which plays an essential role in filtering pathogenic microorganisms.

Alcohol raises the risk of death and worsens the symptoms of ARDS. By increasing alveolar barrier permeability, alcohol consumption affects the oxidative stress response and makes the lungs more sensitive to edematous stress. AUD is often associated with comorbidities such as diabetes mellitus and chronic kidney disease, which also increase the risk for complications in COVID-19 [34].

\section{Conclusion}

Alcohol has an effect on practically every lung cell. Most of these modifications may increase the likelihood of COVID-19 infection 
and more severe pneumonia or ARDS in heavy drinkers. More research is needed to better understand how COVID-19 can benefit people with alcohol use problems. As the epidemic worsens, increased awareness for signs of alcohol withdrawal, as well as rigorous screening of hospitalised patients, will be critical.

In obese patients, alcohol consumption, particularly heavy drinking, is linked to a higher risk of poor COVID-19 clinical outcomes.

\section{Acknowledgements}

I thank my Chief Execute Director who has encouraged us to work on Reviewing on interesting Articles.

\section{Conflict of Interest}

No conflict of interest exists.

\section{Bibliography}

1. Tsatsakis A., et al. "SARS-CoV-2 pathophysiology and its clinical implications: An integrative overview of the pharmacotherapeutic management of COVID-19". Food and Chemical Toxicology 146 (2020): 111769.

2. Calina Daniela., et al. "COVID-19 pandemic and alcohol consumption: Impacts and interconnections". Toxicology Reports 8 (2021): 529-535.

3. Tsatsakis Aristidis., et al. "COVID-19, an opportunity to reevaluate the correlation between long-term effects of anthropogenic pollutants on viral epidemic/pandemic events and prevalence". Food and Chemical Toxicology: An International Journal published for the British Industrial Biological Research Association 141 (2020): 111418.

4. Sharifi-Rad M., et al. "Impact of Natural Compounds on Neurodegenerative Disorders: From Preclinical to Pharmacotherapeutics". Clinical Medicine 90.4 (2020): 1061.

5. Calina Daniela., et al. "COVID-19 pandemic and alcohol consumption: Impacts and interconnections". Toxicology Reports 8 (2021): 529-535.

6. Rehm, Jürgen., et al. "Alcohol use in times of the COVID 19: Implications for monitoring and policy". Drug and Alcohol Review 39.4 (2020): 301-304.

7. Tsatsakis A., et al. "A Mechanistic and Pathophysiological Approach for Stroke Associated with Drugs of Abuse". Journal of Clinical Medicine 8.9 (2019).
8. Salehi Bahare., et al. "Epibatidine: A Promising Natural Alkaloid in Health". Biomolecules 9.1 (2018): 6.

9. Georgakouli Kalliopi., et al. "Effects of acute exercise on liver function and blood redox status in heavy drinkers". Experimental and Therapeutic Medicine 10.6 (2015): 2015-2022.

10. Michalodimitrakis M N., et al. "Suicide by alcohol overdose". Journal of Clinical Forensic Medicine 4.2 (1997): 91-94.

11. Laura nussbaum., et al. "Modern treatment approaches in psychoses. pharmacogenetic, neuroimagistic and clinical implications". Farmacia 65 (2017) 75-81.

12. Pennisi Manuela., et al. "SARS-CoV-2 and the Nervous System: From Clinical Features to Molecular Mechanisms". International Journal of Molecular Sciences 21.15 (2020): 5475.

13. Taylor and Francis Group. "New study shows every week of lockdown increases binge drinking". ScienceDaily. ScienceDaily, 7 December (2020).

14. Grossman Elyse R., et al. "Alcohol Consumption during the COVID-19 Pandemic: A Cross-Sectional Survey of US Adults". International Journal of Environmental Research and Public Health 17.24 (2020): 9189.

15. Coronavirus disease (COVID-19) outbreak [online information portal]. Copenhagen: WHO Regional Office for Europe (2020).

16. Mehrpour Omid., et al. "Toll of acute methanol poisoning for preventing COVID-19". Archives of Toxicology 94.6 (2020): 2259-2260.

17. Dubey Souvik., et al. "Psychosocial impact of COVID-19". Diabetes and Metabolic Syndrome 14.5 (2020): 779-788.

18. Matei Valentin., et al. "Knowledge of Prevention Measures and Information About Coronavirus in Romanian Male Patients with Severe Mental Illness and Severe Alcohol Use Disorder". Neuropsychiatric Disease and Treatment 16 (2020): 28572864.

19. Ram A Sharma, MD., et al. "Alcohol Withdrawal Rates in Hospitalized Patients During the COVID-19 Pandemic". JAMA Network Open 4.3 (2021): e210422.

20. Da Ben L., et al. "Coronavirus Disease 2019 Hangover: A Rising Tide of Alcohol Use Disorder and Alcohol-Associated Liver Disease". Hepatology (Baltimore, Md.) 72.3 (2020): 1102-1108. 
21. Rehm Jürgen., et al. "Alcohol use in times of the COVID 19: Implications for monitoring and policy". Drug and Alcohol Review 39.4 (2020): 301-304.

22. de Goeij Moniek C M., et al. "How economic crises affect alcohol consumption and alcohol-related health problems: a realist systematic review". Social Science Medicine 131 (2015): 131-146.

23. Borkenstein R F and H W Smith. "The Breathalyzer and Its Applications". Medicine, Science and the Law 2.1 (1961): 13-22.

24. de Roux Andrés., et al. "Impact of alcohol abuse in the etiology and severity of community-acquired pneumonia”. Chest 129.5 (2006): 1219-1225.

25. Wyatt Todd A., et al. "Alcohol potentiates RSV-mediated injury to ciliated airway epithelium". Alcohol (Fayetteville, N.Y.) 80 (2019): 17-24.

26. World Health Organization; Alcohol and Covid 19: What you need to know (2020).

27. National Institute on Alcohol Abuse and Alcoholism; Alcohol and covid 19 (2020).

28. Wyatt T A and J H Sisson. "Chronic ethanol downregulates PKA activation and ciliary beating in bovine bronchial epithelial cells". American Journal of Physiology. Lung Cellular and Molecular Physiology 281.3 (2001): L575-581.

29. Bailey Kristina L., et al. "Oxidative stress associated with aging activates protein kinase $\mathrm{C} \varepsilon$, leading to cilia slowing". American Journal of Physiology. Lung Cellular and Molecular Physiology 315.5 (2018): L882-L890.

30. Jain Vageesh and Jin-Min Yuan. "Predictive symptoms and comorbidities for severe COVID-19 and intensive care unit admission: a systematic review and meta-analysis". International Journal of Public Health 65.5 (2020): 533-546.

31. Nihlén Ulf., et al. "Alcohol-induced upper airway symptoms: prevalence and co-morbidity". Respiratory Medicine 99.6 (2005): 762-769.

32. Esper A., et al. "The effect of alcohol abuse on ARDS and multiple organ dysfunction". Minerva Anestesiologica 72.6 (2006): 375-381.
33. Fan Xiude., et al. "Alcohol Consumption Is Associated with Poor Prognosis in Obese Patients with COVID-19: A Mendelian Randomization Study Using UK Biobank". Nutrients 13.5 (2021): 1592.

34. Kushner Tatyana and John Cafardi. "Chronic Liver Disease and COVID-19: Alcohol Use Disorder/Alcohol-Associated Liver Disease, Nonalcoholic Fatty Liver Disease/Nonalcoholic Steatohepatitis, Autoimmune Liver Disease, and Compensated Cirrhosis". Clinical Liver Disease 15.5 (2020): 195-199.

\section{Volume 5 Issue 10 October 2021}

(C) All rights are reserved by Lakshmi Jyothi Tadi., et al. 